\title{
Kagomé Lattice Antiferromagnet Stripped to Its Basics
}

\author{
P. Azaria ${ }^{1}$, C. Hooley ${ }^{2}$, P. Lecheminant ${ }^{3}$, C. Lhuillier ${ }^{1}$, and A. M. Tsvelik ${ }^{2}$ \\ ${ }^{1}$ Laboratoire de Physique Théorique des Liquides, \\ Université Pierre et Marie Curie, 4 Place Jussieu, 75252 Paris, France \\ 2 Department of Physics, University of Oxford, 1 Keble Road, Oxford OX1 3NP, UK \\ ${ }^{3}$ Laboratoire de Physique Théorique et Modélisation, \\ Université de Cergy-Pontoise, Site de Saint Martin, 2 avenue Adolphe Chauvin, 95302 Cergy-Pontoise Cedex, France
}

(Received: )

\begin{abstract}
We study a model of a spin $\mathrm{S}=1 / 2$ Heisenberg antiferromagnet on a one dimensional lattice with the local symmetry of the two dimensional kagomé lattice. Using three complementary approaches, it is shown that the low energy spectrum can be described by two critical Ising models with different velocities. One of these velocities is small, leading to a strongly localized Majorana fermion. These excitations are singlet ones whereas the triplet sector has a spectral gap.
\end{abstract}

PACS No: 75.10.Jm, 75.40.Gb

The famous kagomé lattice antiferromagnet still remains largely a mystery after a decade of extensive studies. This system exhibits both frustration and low coordinance and classically it has infinite continuous degeneracies. Local distortions allow it to explore its many ground states with no cost in energy and lead to a very specific linear spin wave spectrum with a whole branch of zero energy excitations [1]. In the quantum case $(S=1 / 2)$, the system is likely to be a spin liquid with a gap for the magnetic excitations [2]. For finite samples, the system has a huge number of singlet states below the first triplet [3] which is a rather unexpected feature for a twodimensional quantum antiferromagnet. Moreover, the analysis of the specific heat shows the existence of unusual low-lying excitations 4. The presence of these low-lying singlet excitations below the spin gap gives a picture of an intriguing spin liquid that deserves understanding from a general point of view.

In this paper, we shall study a one-dimensional model of spin $\mathrm{S}=1 / 2$ Heisenberg antiferromagnet on a lattice presented on Fig. 1 which retains the local symmetry of the kagomé net. Insight into the behavior of the twodimensional kagomé antiferromagnet might be gained by investigating this simplified system. In particular, the one dimensional model may help us to identify the slow degrees of freedom of the problem.

According to the conventional wisdom the model we study may be viewed as a version of a three-leg spin ladder which in the low-energy limit one should expect to fall into the universality class of the $\mathrm{S}=1 / 2$ Heisenberg spin chain. The latter means that the low-lying excitations (often called spinons) are represented by one gapless bosonic mode (in the language of the theory of critical phenomena this means that the central charge of the model is equal to $\mathrm{C}=1$ [5]). However, the frustration may play its tricks and, as we shall see, a somewhat different scenario is realized. Namely, the bosonic mode decouples into two modes of real (Majorana) fermions $(\mathrm{C}=1 / 2$ each) having different spectra.

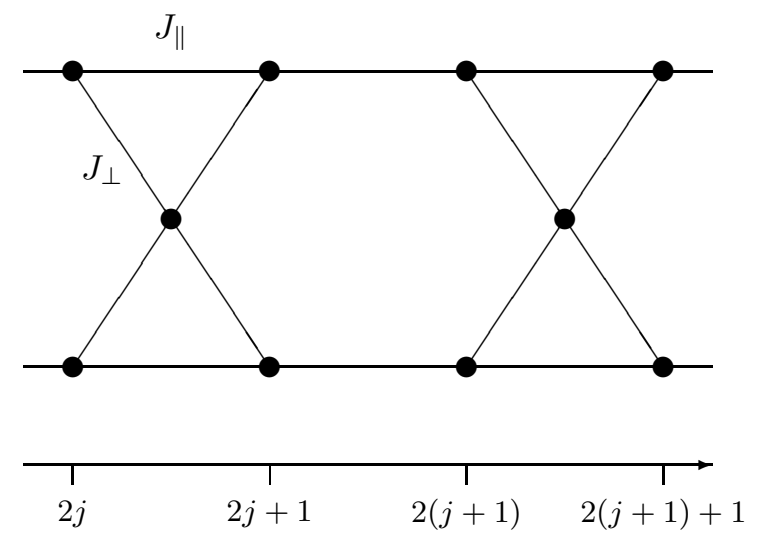

FIG. 1. One dimensional version of the kagomé lattice.

The Hamiltonian for the model shown in Fig. 1 may be written

$$
\begin{array}{r}
\mathcal{H}=\sum_{a=1,2} \sum_{j}\left[J_{\|}\left(\mathbf{S}_{a, 2 j} \cdot \mathbf{S}_{a, 2 j+1}+\mathbf{S}_{a, 2 j+1} \cdot \mathbf{S}_{a, 2 j+2}\right)\right. \\
\left.+J_{\perp} \mathbf{S}_{2 j+\frac{1}{2}} \cdot\left(\mathbf{S}_{a, 2 j}+\mathbf{S}_{a, 2 j+1}\right)\right]
\end{array}
$$

where $\mathbf{s}_{2 j+\frac{1}{2}}$ are the "middle spins", and $\mathbf{S}_{a, j}$ are the "rail spins", the index $a$ taking two values $(a=1,2)$, one for each rail. All interactions are antiferromagnetic and we shall also consider the case where interaction between spins belonging to the rails of the lattice is much stronger than the interactions with the middle spins: $J_{\|}>>J_{\perp}$. Under this condition, one can describe the chains in the continuous approximation, representing the spins on each chain as a sum of ferromagnetic $\left(\mathbf{M}_{a}\right)$ and staggered $\left(\mathbf{n}_{a}\right)$ parts: $\mathbf{S}_{a, j} \rightarrow \mathbf{M}_{a}(x)+(-1)^{j} \mathbf{n}_{a}(x)$. It is crucial for our analysis that the middle spins interact only with the ferromagnetic part of the magnetization. The latter has the following remarkable property - it can be written as a sum of "currents" $\mathbf{M}_{a}=\mathbf{J}_{a}+\overline{\mathbf{J}}_{a}$, where the currents satisfy the same commutation relations as bilinear combinations of left- and right-moving Dirac fermions [6]: 


$$
\overline{\mathbf{J}}_{a}=R_{a, \alpha}^{+} \vec{\sigma}_{\alpha \beta} R_{a, \beta} / 2, \mathbf{J}_{a}=L_{a, \alpha}^{+} \vec{\sigma}_{\alpha \beta} L_{a, \beta} / 2
$$

where $\vec{\sigma}$ are the Pauli matrices. The corresponding algebra $\left(\mathrm{SU}(2)_{1}\right)$ is called the level $k=1 \mathrm{SU}(2)$ Kac-Moody (KM) algebra where $k$ refers to the number of species of spin- $1 / 2$ fermions (one in the given case). Now we notice that the interaction includes the sum of two $\mathrm{SU}(2)_{1}$ spin currents $\mathbf{J}=\mathbf{J}_{1}+\mathbf{J}_{2}$ which, by definition, is a level $k=2$ current. These currents have the same commutation relations as currents of three Majorana fermions (the explicit expression is given later) [7,8]. Since each Dirac fermion can be represented as a linear combination of two Majorana (real) fermions, it is clear that only $3 / 2$ of the low-energy degrees of freedom of rails are involved in the interaction. Obviously the other decoupled degrees of freedom remain critical. Using the results of Ref. [8] we can represent the continuous limit of the Hamiltonian (11) as follows: $\mathcal{H}=\mathcal{H}_{s}+\mathcal{H}_{t}$ with

$$
\begin{array}{r}
\mathcal{H}_{s}=-\frac{\mathrm{i} v}{2} \int \mathrm{d} x\left(r_{0} \partial_{x} r_{0}-l_{0} \partial_{x} l_{0}\right) \\
\mathcal{H}_{t}=\frac{\pi v}{2} \int \mathrm{d} x\left[\mathbf{J}^{2}(x)+\overline{\mathbf{J}}^{2}(x)\right] \\
+\sum_{j} g \int \mathrm{d} x \delta(x-a j) \mathbf{s}_{j} \cdot[\mathbf{J}(x)+\overline{\mathbf{J}}(x)] .
\end{array}
$$

Here $v \sim J_{\|}$is the spin velocity which we shall later put to unity and the coupling constant is $g \sim J_{\perp}$. The Majorana fermion $\left(r_{0}, l_{0}\right)$ describes non-magnetic, gapless excitation. It represents Ising degrees of freedom that do not interact with the central spins and are associated with a discrete $Z_{2}$ interchange symmetry between the surface chains. We shall study the Hamiltonian (位) using several alternative approaches. First, we shall study an integrable deformation of model (四) . To make sure that the exact solution reproduces the qualitative features of the spectrum, we shall consider an anisotropic version of (4) bearing in mind that it may simplify in the limit of strong anisotropy (the so-called Emery-Kivelson limit [9]). Finally, a direct mean field treatment of the lattice in the isotropic limit will be performed.

Exact solution of a related model. Since the currents satisfying the level $k=2 \mathrm{SU}(2) \mathrm{KM}$ algebra can be represented as fermionic bilinears, the effective theory for the central spins corresponds to a special version of the two-channel $(k=2$ !) Kondo-lattice model where "electrons" do not experience backscattering. A similar model (with $k=1$ ) was considered in Ref. [10]; the result is that integrability is achieved if one adds an additional interaction:

$$
\mathcal{H}_{e x}=\mathcal{H}_{t}+g^{\prime} \int \mathrm{d} x \mathbf{J}(x) \cdot \overline{\mathbf{J}}(x)
$$

where the ratio $g^{\prime} / g$ is fixed. The Bethe ansatz equations for model (5) are derived in the same way as in Ref. [10] with the only difference that "conduction electrons" now belong to the spin-1 representation of the $\mathrm{SU}(2)$ group. The result is

$$
\left[e_{2}\left(x_{a}+1 / g\right) e_{2}\left(x_{a}-1 / g\right)\right]^{N} e_{1}^{N_{0}}\left(x_{a}\right)=\prod_{b=1}^{M} e_{2}\left(x_{a}-x_{b}\right)
$$

where $2 N$ and $N_{0}$ are the numbers of spins on the rails and in the middle, respectively, $M$ is the number of up spins $\left(M=2 N+N_{0} / 2-S^{z}\right)$ and $e_{n}(x)=(x-\mathrm{i} n / 2) /(x+$ in/2). The total energy takes the form

$$
E=\frac{1}{2 \mathrm{i}} \sum_{a} \ln \left[e_{2}\left(x_{a}+1 / g\right) / e_{2}\left(x_{a}-1 / g\right)\right] .
$$

Following the standard procedures of the Bethe ansatz, we derive the following system of integral equations for the free energy:

$$
\begin{aligned}
F & =-N T \int \mathrm{d} x[s(x+1 / g)+s(x-1 / g)] \ln \left[1+\mathrm{e}^{\epsilon_{2}(x) / T}\right] \\
& -N_{0} T \int \mathrm{d} x s(x) \ln \left[1+\mathrm{e}^{\epsilon_{1}(x) / T}\right],
\end{aligned}
$$

with

$$
\begin{aligned}
\epsilon_{j}(x)=T s * \ln \left[1+\mathrm{e}^{\epsilon_{j-1}(x) / T}\right][ & \left.1+\mathrm{e}^{\epsilon_{j+1}(x) / T}\right] \\
& -\delta_{j, 2} \Delta \cosh \pi x
\end{aligned}
$$

and $\lim _{n \rightarrow \infty} \epsilon_{n}(x) / n=H, H$ being the magnetic field. In (9), one has $\Delta \sim \exp (-\pi / g), s(x)=[2 \cosh \pi x]^{-1}$, and the convolution product is denoted by $*$.

At low temperatures $T<<\Delta$, one has $\epsilon_{2}(x) \approx$ $-\Delta \cosh \pi x, \quad \epsilon_{1}(x)=O\left(\mathrm{e}^{-\Delta / T}\right)$. In this case the first term in the free energy (8) gives an exponentially small contribution corresponding to excitations with a spectral gap $\Delta$ and the last term gives $F \approx-T N_{0} \ln \sqrt{2}$ showing that each central spin contributes $\ln \sqrt{2}$ to the ground state entropy. A lesson we learn from the exact solution is that there are degrees of freedom presumably localized on central spins (and their number corresponds exactly to a single Majorana mode) which remain decoupled. One may conjecture that since the magnetic modes are separated from the ground state by a gap, the soft modes will remain soft even when one departs from the integrable point. Below we shall give more rigorous arguments to support this conjecture.

The Emery-Kivelson limit. We shall now study a U(1) version of the Hamiltonian (4), characterized by anisotropic interactions with $g \rightarrow g_{\|}, g_{\perp}$ where in the limit of strong anisotropy, the low energy degrees of freedom can be identified. This approach has been fruitfully applied in quantum impurity problems and gives a simple description of the two-channel Kondo model [9] and also of the Kondo lattice [11]. In particular, for the twochannel Kondo problem, Emery-Kivelson identified the residual zero point entropy stemming from a unique Majorana zero mode. To reformulate the Hamiltonian (位) as a fermionic theory similar to Kondo problems, we first introduce three right- and left-moving Majorana fermions 
$r_{b}, l_{b}(b=1,2,3)$ to use the fermionic representation of the $\mathrm{SU}(2)_{2}$ spin current: $\bar{J}^{a}=-\mathrm{i} \epsilon^{a b c} r_{b} r_{c} / 2$ with a similar relation for the left-moving current [7]. The next step in our solution is to combine the two Majorana fermions $\left(r_{1}, l_{1}\right)$ and $\left(r_{2}, l_{2}\right)$ to form a single Dirac spinor $(R, L)$ which in turn can be bosonized. Introducing a massless bosonic field $\Phi$ and its dual field $\Theta$, one can write:

$$
\begin{gathered}
R=\frac{r_{1}+\mathrm{i} r_{2}}{\sqrt{2}}=\frac{\epsilon}{\sqrt{2 \pi a_{0}}} \exp [\mathrm{i} \sqrt{\pi}(\Phi-\Theta)] \\
L=\frac{l_{1}+\mathrm{i} l_{2}}{\sqrt{2}}=\frac{\epsilon}{\sqrt{2 \pi a_{0}}} \exp [-\mathrm{i} \sqrt{\pi}(\Phi+\Theta)]
\end{gathered}
$$

where $a_{0}$ is a short distance cut-off. The anticommutation relation between $R$ and $L$ is taken into account by the commutator: $[\Theta(y), \Phi(x)]=-\mathrm{i} \theta(y-x)$. The real fermionic zero mode $(\epsilon)$ is necessary to establish the correct anticommutation relations with the third Majorana fermion $\left(r_{3}, l_{3}\right)$. The interacting part of the Hamiltonian (蛋) is then given by:

$$
\begin{array}{r}
\mathcal{H}_{\text {int }}=\sum_{j}\left(-\frac{g_{\|}}{\sqrt{\pi}} s_{j}^{z}\left(\partial_{x} \Phi\right)_{j}+\frac{g_{\perp}}{\sqrt{4 \pi a_{0}}} \epsilon_{j} s_{j}^{+} \mathrm{e}^{i \sqrt{\pi} \Theta_{j}}\right. \\
\left.\left(\mathrm{e}^{i \sqrt{\pi} \Phi_{j}} \mathrm{e}^{-i \pi / 4} l_{3, j}+\mathrm{e}^{-i \sqrt{\pi} \Phi_{j}} \mathrm{e}^{i \pi / 4} r_{3, j}\right)+h . c\right) .
\end{array}
$$

Following Emery and Kivelson, we absorb the phase factor $\mathrm{e}^{ \pm i \sqrt{\pi} \Theta_{j}}$ into the spin operators by a unitary transformation $U$ :

$$
U=\mathrm{e}^{-i \sqrt{\pi} \sum_{j} \Theta_{j} s_{j}^{z}}
$$

As a result the interaction becomes

$$
\begin{array}{r}
\mathcal{H}_{i n t} \rightarrow U \mathcal{H}_{i n t} U^{\dagger}=\sum_{j}\left(-\frac{\left(g_{\|}-\pi\right)}{\sqrt{\pi}} s_{j}^{z}\left(\partial_{x} \Phi\right)_{j}\right. \\
\left.+\frac{g_{\perp}}{\sqrt{4 \pi a_{0}}} a_{j}^{+}\left(\mathrm{e}^{i \sqrt{\pi} \Phi_{j}} l_{3, j}+(-1)^{j} \mathrm{e}^{-i \sqrt{\pi} \Phi_{j}} r_{3, j}\right)+\text { h.c. }\right)
\end{array}
$$

where we have replaced the combinations of spin- $1 / 2$ operators and fermionic zero mode by local fermions $\left(a_{j}\right)$ using the Jordan-Wigner transformation. We have also absorbed a phase factor $(-\mathrm{i})^{j}$ in the definition of $a_{j}^{+}$.

At a special point $g_{\|}=\pi$ (called the Toulouse point), part of the interaction vanishes and the low energy physics can be studied by a simple mean-field theory. Since the scaling dimension $d$ of the bosonic exponents in (13) is pretty small $(d=1 / 4)$, we shall replace them by their averages and try to solve the problem selfconsistently. Introducing two Majorana fermions $\left(\xi_{1,2}\right)$ associated with the complex fermion $a: a=\left(\xi_{1}+\mathrm{i} \xi_{2}\right) / \sqrt{2}$, we find that the effective action decouples into bosonic and fermionic parts in the mean field limit: $\mathcal{S}_{M F}=$ $\mathcal{S}_{B}+\mathcal{S}_{F}$ with

$$
\mathcal{S}_{B}=\int d x d \tau\left[\frac{1}{2}\left(\partial_{\mu} \Phi\right)^{2}+\lambda \cos (\sqrt{\pi} \Phi)\right]
$$

$$
\begin{array}{r}
\mathcal{S}_{F}=\sum_{j} \int d \tau\left[\frac{1}{2} \xi_{1, j} \partial_{\tau} \xi_{1, j}+\frac{1}{2} \xi_{2, j} \partial_{\tau} \xi_{2, j}\right. \\
\left.+\frac{1}{2} \chi_{j} \partial_{\tau} \chi_{j}-\mathrm{i} \chi_{j+1} \chi_{j}-\mathrm{i} \Delta \xi_{2, j} \chi_{j}\right]
\end{array}
$$

where $\chi$ is a Majorana fermion express in terms of the right- and left-moving Majorana fields $\left(r_{3}, l_{3}\right)$ : $\chi_{j}=\left(l_{3, j}+(-1)^{j} r_{3, j}\right) / \sqrt{2}$. The mean-field parameters are defined by: $\lambda=-\mathrm{i} g_{\perp}\left\langle\xi_{2} \chi\right\rangle / \sqrt{\pi a_{0}}$ and $\Delta=$ $g_{\perp}\langle\cos (\sqrt{\pi} \Phi)\rangle / \sqrt{\pi a_{0}}$. Solving the self-consistency equations, we find that the bosonic field $\Phi$ becomes massive with a gap of the order $\Delta \sim g_{\perp}^{4 / 3} \ln ^{1 / 6}\left(1 / g_{\perp}^{2} a_{0}\right)$. In the fermionic sector, the Majorana fermion $\xi_{2}$ hybridizes with the Majorana field $\chi$ which in terms of the original spins stems from the rails spins. The resulting excitation spectrum is reminiscent of the one found in Kondo lattices [12] with a small gap $\Delta_{g} \sim \Delta^{2} / J_{\|} \ll \Delta$. Finally, there is still a singlet localized Majorana fermion $\xi_{1}$ which decouples from the conduction sea at the Toulouse point. This mode gives a zero point entropy of magnitude $\frac{1}{2} \ln 2$ per central spin as found above in the integrable model. Away from the Toulouse point $\left(\delta g_{\|}=g_{\|}-\pi \neq 0\right)$, this mode will acquire a small dispersion and will contribute to the specific heat. Coming back to the original model, adding the contribution of the singlet Majorana fermion $\left(r_{0}, l_{0}\right)$ which has decoupled from the interaction, the total central charge in the long-distance limit is $\mathrm{C}=1$. Let us stress that the two Majorana modes, in the singlet sector, contributing to the central charge are of different nature. The Majorana field $\left(r_{0}, l_{0}\right)$ describes a critical Ising model whereas $\xi_{1}$ is a strongly localized Majorana fermion. In contrast, the triplet sector has a small spectral gap. As a result of this spectrum, the middle spins are disordered and have short-ranged spin correlations.

The experience gained from the study of Kondo models leads us to expect that the preceeding results obtained in the Emery-Kivelson limit of the model will extend to the isotropic point. To show this, we shall now develop a mean-field theory directly at the isotropic limit keeping track of the lattice structure more accurately than in the previous approach.

Direct mean-field approach. The Hamiltonian (4) is reformulated in terms of a fermionic model on the lattice:

$$
\begin{array}{r}
\mathcal{H}_{t}=\mathrm{i} J_{\|} \sum_{j} \chi_{j+1}^{a} \chi_{j}^{a}-\frac{i g}{2} \sum_{j} \mathbf{s}_{2 j+\frac{1}{2}} \cdot\left(\left[\vec{\chi}_{2 j} \times \vec{\chi}_{2 j}\right]\right. \\
\left.+\left[\vec{\chi}_{2 j+1} \times \vec{\chi}_{2 j+1}\right]\right) .
\end{array}
$$

In the continuum limit, the rail of $\chi^{a}$ fermions will contain left- and right- moving Majorana fermions $\left(r_{a}, l_{a} ; a=1,2,3\right)$ of the $\mathrm{SU}(2)_{2}$ spin current: $\chi_{j}^{a}=$ $-l_{a}(x)-(-1)^{j} r_{a}(x)$; with this identification, the Hamiltonian (4) is then reproduced. The model (15) corresponds to local moments $\left(\mathbf{s}_{2 j+1 / 2}\right)$ interacting with a sea of three Majorana fermions $\left(\chi_{j}^{a}\right)$. To describe the middle spins we use the Majorana representation for spins $\mathrm{S}=1 / 2$ (see Ref. 12 and references therein): 


$$
\mathbf{s}_{n}=-\frac{\mathrm{i}}{2}\left[\vec{\gamma}_{n} \times \vec{\gamma}_{n}\right], \quad n=2 k+\frac{1}{2}, k \in \mathcal{Z}
$$

where $\gamma_{n}^{a}$ are local Majorana fermions satisfying the anticommutation relations $\left\{\gamma_{n}^{a}, \gamma_{m}^{b}\right\}=\delta_{n m} \delta^{a b}$. This representation (16) reproduces the spin commutation relations and gives the correct value of the Casimir operator: $\mathbf{s}_{n}^{2}=3 / 4$.

A model very similar to (15) was analyzed in Ref. [13. Following this analysis we substitute (16) into (15) and decouple the interaction with an auxiliary field $V$ living on the links connecting rails with middle spins. In the mean-field approximation the variables $V$ are considered as static with their values determined self-consistently by minimizing the free energy. We find that the minimum is achieved when a unit cell contains two middle spins (see Fig. 2) with either $U_{+}=V_{-}=0, U_{-}= \pm V_{+} \equiv \Delta$ or $U_{-}=V_{+}=0, U_{+}= \pm V_{-} \equiv \Delta$.

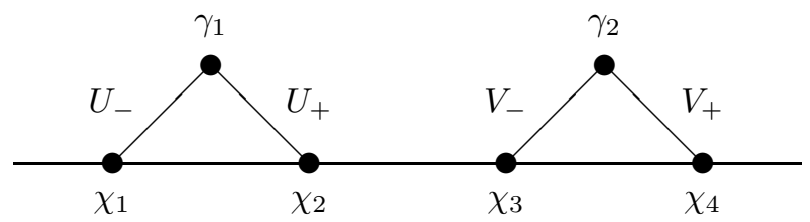

FIG. 2. The unit cell in the mean-field approximation.

All branches of the spectrum are found to be gapful. The low energy band is rather flat towards the edge of the Brillouin zone in agreement with the Emery-Kivelson limit of the model in the triplet sector. However, as was demonstrated in Ref. [13], the local $\mathrm{Z}_{2}$-degeneracy of the ground state generates a local real fermionic zero mode $\gamma_{0}$ which is coupled to the three Majorana band fermions with the Lagrangian:

$$
L=\sum_{j}\left[\frac{1}{2} \gamma_{j}^{0} \partial_{\tau} \gamma_{j}^{0}+g^{\prime} \gamma_{j}^{0} \chi_{j}^{1} \chi_{j}^{2} \chi_{j}^{3}\right] .
$$

This singlet zero mode acquires a small dispersion with the bandwidth $\Delta_{g} \sim \Delta^{2} / J_{\|} \ll \Delta$. The total amount of entropy accumulated in this band is $\frac{1}{2} \ln 2$ per central spin and this degeneracy will be slightly lifted and results in the coherent dispersion of this mode. This mode corresponds to the strongly localized Majorana fermion found in the Emery-Kivelson limit. This field will manifest itself in the spin-spin correlation functions of the central spins since at low energies, they behave like $\mathbf{s} \sim \gamma_{0} \vec{\chi}$. Due to the small dispersion of the fermionic mode $\gamma_{0}$, correlation functions of middle spins are strongly localized in space, but not in time where the characteristic scale is $\sim \Delta_{g}^{-1}$ :

$$
\langle\langle\mathbf{s}(\tau, j) \mathbf{s}(0, j)\rangle\rangle \sim K_{1}\left(\Delta_{g}|\tau|\right)
$$

which for small times is proportional to $1 /|\tau|$, like for the two-channel Kondo problem.
In conclusion, the version of the kagomé lattice studied in this paper can be reformulated as a fermionic theory similar to models of Kondo lattices. Its low-energy excitations are two spin-singlet Majorana modes with different spectra: a critical Ising mode and a strongly localized Majorana fermion, whereas the triplet sector has a small spectral gap. The physics of the localized low-energy mode is similar to physics of the two-channel Kondo model. This picture retains some properties of the kagomé antiferromagnet with very soft singlet modes and a gap for magnetic excitations. It is tempting to conjecture that the singlet degrees of freedom and the gapful magnetic excitation identified here might be responsible for the additional structure seen in the specific heat of the kagomé magnet at low temperature.

A. M. Tsvelik acknowledges the kind hospitality of Ecole Normale Supérieure during his stay in Paris. The authors thank P. Chandra, P. Coleman, B. Douçot, H.-U. Everts, and Ch. Waldtmann for important discussions.

[1] J. T. Chalker, P. C. W. Holdsworth, and E. S. Shender, Phys. Rev. Lett. 68, 855 (1992); A. B. Harris, C. Kallin, and A. J. Berlinsky, Phys. Rev. B 45, 2899 (1992); A. Chubukov, Phys. Rev. Lett. 69, 832 (1992); P. Chandra, P. Coleman, and I. Ritchey, J. Phys. I France 3, 591 (1993).

[2] C. Zeng and V. Elser, Phys. Rev. B 42, 8436 (1990); J. T. Chalker and J. F. G. Eastmond, Phys. Rev. B 46, 14201 (1992); R. R. P. Singh and D. A. Huse, Phys. Rev. Lett. 68, 1766 (1992); S. Sachdev, Phys. Rev. B 45, 12 377 (1992); P. W. Leung and V. Elser, Phys. Rev. B 47, 5459 (1993).

[3] P. Lecheminant, B. Bernu, C. Lhuillier, L. Pierre, and P. Sindzingre, Phys. Rev. B 56, 2521 (1997).

[4] N. Eltsner and A.P. Young, Phys. Rev. B 50, 6871 (1994).

[5] G. Sierra, J. Phys. A 29, 3299 (1996).

[6] I. Affleck, Nucl. Phys. B265, 409 (1986).

[7] A. B. Zamolodchikov and. V. A. Fateev, Sov. J. Nucl. Phys. 43, 657 (1986).

[8] D. G. Shelton, A. A. Nersesyan, and A. M. Tsvelik, Phys. Rev. B 53, 8521 (1996); D. Allen and D. Sénéchal, Phys. Rev. B 55, 299 (1997).

[9] V. J. Emery and S. A. Kivelson, Phys. Rev. B 46, 10812 (1992).

[10] E. H. Rezayi and J. Sak, Phys. Lett. 89A, 451 (1982).

[11] O. Zachar, S. A. Kivelson, and V. J. Emery, Phys. Rev. Lett. 77, 1342 (1996); P. Coleman, A. Georges, and A. M. Tsvelik, J. Phys. Cond. Matt. 9, 345 (1997).

[12] A. M. Tsvelik, Phys. Rev. Lett. 69, 2142 (1992); P. Coleman, E. Miranda, and A. M. Tsvelik, Phys. Rev. B 49, 8955 (1994).

[13] P. Coleman, L. B. Ioffe, and A. M. Tsvelik, Phys. Rev. B 52, 6611 (1995). 